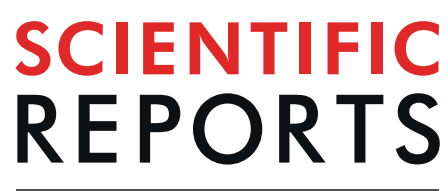

natureresearch

Check for updates

\title{
Formation of gigahertz pulse train by chirped terahertz pulses interference
}

\author{
Xinrui Liu ${ }^{1}$, Maksim Melnik ${ }^{1}$, Maria Zhukova ${ }^{1}{ }^{凶}$, Egor Oparin $^{1}$, Joel J. P. C. Rodrigues ${ }^{1,2,3}$, \\ Anton Tcypkin ${ }^{1} \&$ Sergei Kozlov ${ }^{1}$
}

The state-of-art broadband THz sources can contribute to the development of short-range 6G communications. This paper has demonstrated the feasibility of forming the controllable sequence of THz subpulses in the temporal domain and the corresponding quasidiscrete spectrum by the interference of two THz pulses with an exponential chirp. Moreover, due to small time delay between these pulses the temporal and spectral structures are similar to each other (so-called "linkage relation"). This will benefit information encoding in the $\mathrm{THz}$ range. The calculated metrics for the prototype communication channel based on the proposed method are competitive with existing short-range $\mathrm{THz}$ CW channels.

The maturity and commercialization of the fifth generation $(5 \mathrm{G})$ has already arrived ${ }^{1}$. $6 \mathrm{G}$ (6th generation wireless systems) - a technology that supposed 100 to 1000 times $^{2,3}$ faster than $5 \mathrm{G}$, is considered to come to our lives in the next ten years ${ }^{4}$. Fresh spectral bands as well as advanced physical layer solutions are required for future wireless communications and $\mathrm{THz}$ wireless technology is a potential candidate ${ }^{5-9}$. Usually optical frequency combs are used in IR optical communication systems providing high data rates for long distances ${ }^{10}$. In this case, a large number of channels can be created using spectrum-sliced supercontinuum (SSSC) by arranging the interference of the two pulses with ultra-broadband spectra ${ }^{11,12}$. In the $\mathrm{THz}$ range a typical method to generate $\mathrm{THz}$ spectral comb is $\mathrm{THz}$ generator pumped by a pulse train of fs laser pulses ${ }^{13}$. It has also been reported that the transmitted spectrum of a THz pulse through a multilayer filter could achieve sub-comb structure ${ }^{14}$. As a THz frequency comb, it can be used to measure the frequency of a continuous-wave $\mathrm{THz}$ with high precision ${ }^{15}$; is suitable for the source of $\mathrm{THz}$ wireless communication of wavelength division multiplexing ${ }^{16}$, and expected to be used in ultra-fast information transmission systems ${ }^{17}$. The use of pulsed $\mathrm{THz}$ sources is limited by strong free space losses due to the wide spectrum which does not overlap with atmospheric transparency window ${ }^{18}$. Therefore, THz links have been suggested to use pulsed sources for short distance indoor communications ${ }^{19,20}$, and to use continuous radiation sources for long distances ${ }^{21,22}$. The implementation of these techniques relies on ultra-fast modulation as well as encoding and decoding methods of $\mathrm{THz}$ wave, in which pulsed broadband $\mathrm{THz}$ technologies can make contributions $\mathrm{s}^{23}$.

In the case of pulsed broadband radiation, the main aim is to create encoding methods based on wavelength division multiplexing (WDM). For instance, quasi-discrete $\mathrm{THz}$ supercontinuum, obtained via spectral interference of two THz pulses, can be used to achieve a data transfer rate of $34.1 \mathrm{~Gb} / \mathrm{s}$ with $31 \mathrm{THz}$ spectral lines ${ }^{24}$. In the NIR region, the profiling of the spectral structure and the corresponding pulse sequence with a THz repetition rate has been verified both experimentally and analytically ${ }^{25-27}$. The peculiarity of this approach lies in the fact that there is the pulse train corresponding to the profiling spectral structure ("linkage relation") which helps to preserve transmitted data from corruption. In the case of pulsed $\mathrm{THz}$ radiation, which consists of a small number of oscillations ${ }^{28}$, the formation of these structures by aforementioned method occurs when the following conditions are met: the THz pulses should be carefully chirped and the time delay between them is shorter than their duration. In the $\mathrm{THz}$ range, chirping and pulse elongation can be accomplished by using hollow metal waveguides ${ }^{29}$.

In this paper, we show for the first time the possibility of forming a frequency comb and the corresponding temporal pulses sequence in $\mathrm{THz}$ frequency range. The "linkage relation" between the emerging temporal and spectral structures is shown. Thus, changes in spectrum will lead to similar changes in temporal domain. Using

${ }^{1}$ International Laboratory of Femtosecond Optics and Femtotechnologies, ITMO University, St. Petersburg, 197101, Russia. ${ }^{2}$ Federal University of Piauí, (UFPI), Teresina, Pi, 64049-550, Brazil. ${ }^{3}$ Instituto de Telecomunicações, 1049001, Lisboa, Portugal. ${ }^{凶}$-mail: mozhukova@itmo.ru 


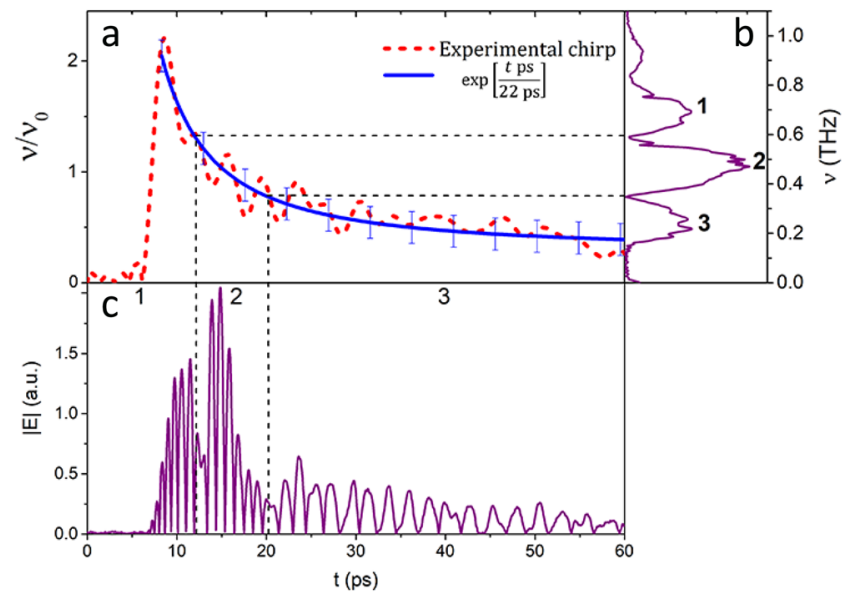

Figure 1. (a) Experimental chirp of THz pulse and its exponential approximation, (b) quasi-discrete spectrum (c) and temporal structure of the pulse train.

proposed method data rate of $225 \mathrm{Mbit} / \mathrm{sec}$ can be achieved. The communication channel based on this method is comparable with existing short-range $\mathrm{THz} \mathrm{CW}$ channels ${ }^{30-33}$. However these results can be improved by implementing pump laser with higher repetition rate and adjusting the interferometer. This technique allows to create short-range communication network and devices which can operate at room temperature.

\section{Results}

The experimental part of the work was carried out on a setup consisting of a THz spectrometer with a Michelson interferometer (see Methods). The interferometer was used to form two fs pulses with a variable delay between them of a longer duration. The obtained fs pulses generated $\mathrm{THz}$ pulses by photo-Dember effect in InAs crystal. THz pulses, in turn, propagated in a hollow metal waveguide chirping and interfering. The presence of the chirp enables to observe not only the formation of a quasi-discrete spectrum ${ }^{24}$, but also the formation of a train of pulses during the interference 26,27 . This is possible due to the correct selection of conditions, namely, a small delay between pulses and the presence of a chirp with a known dependence. The chirp of experimentally obtained THz pulses is shown in Fig. 1(a) (red curve). As can be seen, it is well approximated by an exponential function (blue curve). THz pulse propagation through the waveguide can be described as a linear superposition of the coupled propagating modes. The parameters of the waveguide, as well as the characteristics of the THz radiation, determine the conditions for modes coupling and thus the appearance of the output radiation, for instance, its chirp. More details about physical interpretation can be found in ${ }^{29,34}$. Figure $1(b, c)$ illustrates the experimental quasi-discrete spectrum and the temporal structure of the formed pulse train.

It was previously shown ${ }^{26,27}$ that a linearly chirped pulse interfering with itself shifted by a time delay shorter than its duration formed a pulse train which had a strict corresponding relation to its quasi-discrete spectrum. This means that each subpulse in the temporal structure has its own spectral line in the quasi-discrete spectrum. In this paper, due to the exponential chirp in the $\mathrm{THz}$ spectrum, the presence of the correspondence between the temporal and spectral structures is not obvious. In Fig. 1(b) three discernible pulse spikes can be seen. In the temporal structure Fig. 1(c) these spikes correspond to three pulses of different frequencies and duration. The formation of an irregular temporal sequence is associated with a nonlinear (exponential) pulse chirp. Figure 1(c) shows that the duration of individual peaks in the sequence varies from 10 to $40 \mathrm{ps,}$ which corresponds to a repetition rate of $25-100 \mathrm{GHz}$. In this case, the chirp determines the correlation between the temporal and spectral structures ${ }^{27}$. Moreover, encoding can be carried out in the spectral domain, where we have a regular quasidiscrete structure Fig. 1(b) formed by two-beam interference ${ }^{11}$. Encoding in this case, should be done in the spectral domain by separating the spectrum in space and using amplitude filters, as it was previously shown in NIR range $\mathrm{e}^{26}$.

\section{Discussion}

To verify the assumptions made during the analysis of experimental data, a numerical simulation was carried out with parameters close to experimental ones. $\mathrm{THz}$ pulse with exponential chirp can be represented as:

$$
E=E_{0} \cdot \exp \left(-2 \frac{t^{2}}{\tau_{0}^{2}}\right) \cdot \sin \left(\omega_{0}(1+\exp (-\alpha t)) t\right)
$$

where $E_{0}$ is the pulse amplitude, $\omega_{0}=2 \pi v_{0}$ and $v_{0}$ is the pulse central frequency, $\tau_{0}$ is the pulse duration, $\alpha$ is the inverse steepness of exponential chirp. To match the experiment, these parameters were chosen as follows: $v_{0}=$ $0.45 \mathrm{THz}, \tau_{0}=17 \mathrm{ps}$ and $\alpha=1 / 22 \mathrm{ps}^{-1}$. The difference between pulse duration $\tau_{0}$ in numerical simulation and experiment is caused by the fact that a Gaussian pulse profile is used in the numerical simulation, while in the experiment the chirped $\mathrm{THz}$ pulse has an asymmetric non-Gaussian profile. Therefore, the pulse duration in the numerical simulation was increased to match the experimental pulse duration. 

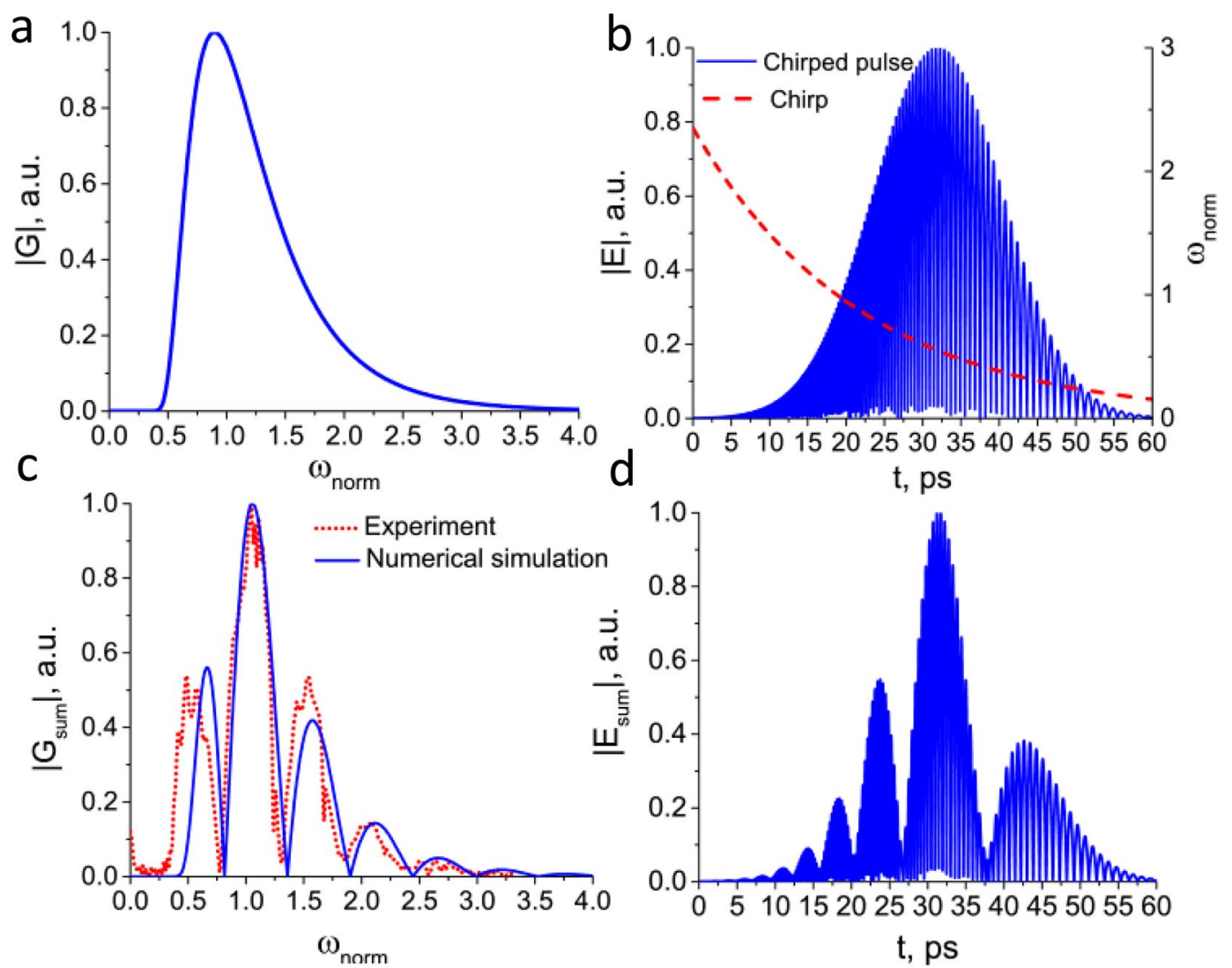

Figure 2. (a) Spectrum, (b) temporal structure and chirp of simulated chirped THz pulse. (c) Quasi-discrete spectrum and (d) pulse train formed from interference of two chirped pulses.

Figure 2(a,b) illustrates spectrum and temporal structure of the exponential chirped $\mathrm{THz}$ pulse obtained from numerical simulation. The interference of two pulses in temporal domain can be represented $\mathrm{as}^{26,27}$ :

$$
E_{\text {sum }}=E(t)+E(t+\Delta t)
$$

where $\Delta t$ is the time delay between pulses. Figure 2(c,d) represents such pulse interference with itself shifted by the time delay $\Delta t=4 \mathrm{ps}$ and the comparing of the corresponding quasi-discrete spectrum with experimental results (Fig. 2(c)). It can be seen that the simulated spectrum agrees well with the experimental one. As already noted, in the numerical simulation a Gaussian profile is used to describe the temporal structure. In the experiment, the temporal structure has an asymmetric non-Gaussian profile. In this regard, a comparison of these structures, as well as their interference is not indicative, since in the experiment asymmetric broadening with respect to the maximum occurs (see Fig. 5(c)), while the numerical simulation gives a symmetric broadening (see Fig. 2(b)).

To confirm the correspondence between the temporal and spectral structures formed during the interference, a numerical simulation of the removal of one line in the quasi-discrete spectrum was performed. The results are shown in Fig. 3. It can be seen that cutting out one of the spectral peaks leads to the vanishing of the subpulse in temporal structure. However, there is some ambiguity in temporal domain which can be explained by the presence of a residual interference term. This term can be eliminated by the proper selection of experimental parameters ${ }^{27}$. Thus, changes in spectrum lead to similar changes in temporal domain. In other words there is a "linkage relation" between spectrum and temporal structure of THz pulse train. It should be noted that in the case of two-beam interference, the position of the minima of the quasidiscrete structure in the spectral region, which is determined by the time delay between pulses, is fixed. The absolute value of modulated spectrum is described by the following expression ${ }^{11}$ :

$$
\left|G_{\text {sum }}(\omega)\right|=\left|G_{0}(\omega)\right|(1+\cos (\omega \Delta t))
$$

where $\Delta t$ is a time delay between pulses and correspond to $3 \mathrm{ps}$ in our experiment. The error associated with a double-pass of interferometer is equal to $2 \mu \mathrm{m}(\sim 6.7 \mathrm{fs})$. Therefore the accuracy of the minima position in the spectrum can be calculated as the ratio of the aforementioned value to the delay between pulses to be $0.2 \%$. In addition, as mentioned above encoding and decoding take place in the spectral range. The positions of spectral minima are fixed, and will not be affected by the instability of driving laser energy and its duration that leads to the changes in the temporal domain. As a result, this will not affect the transmitted information. 

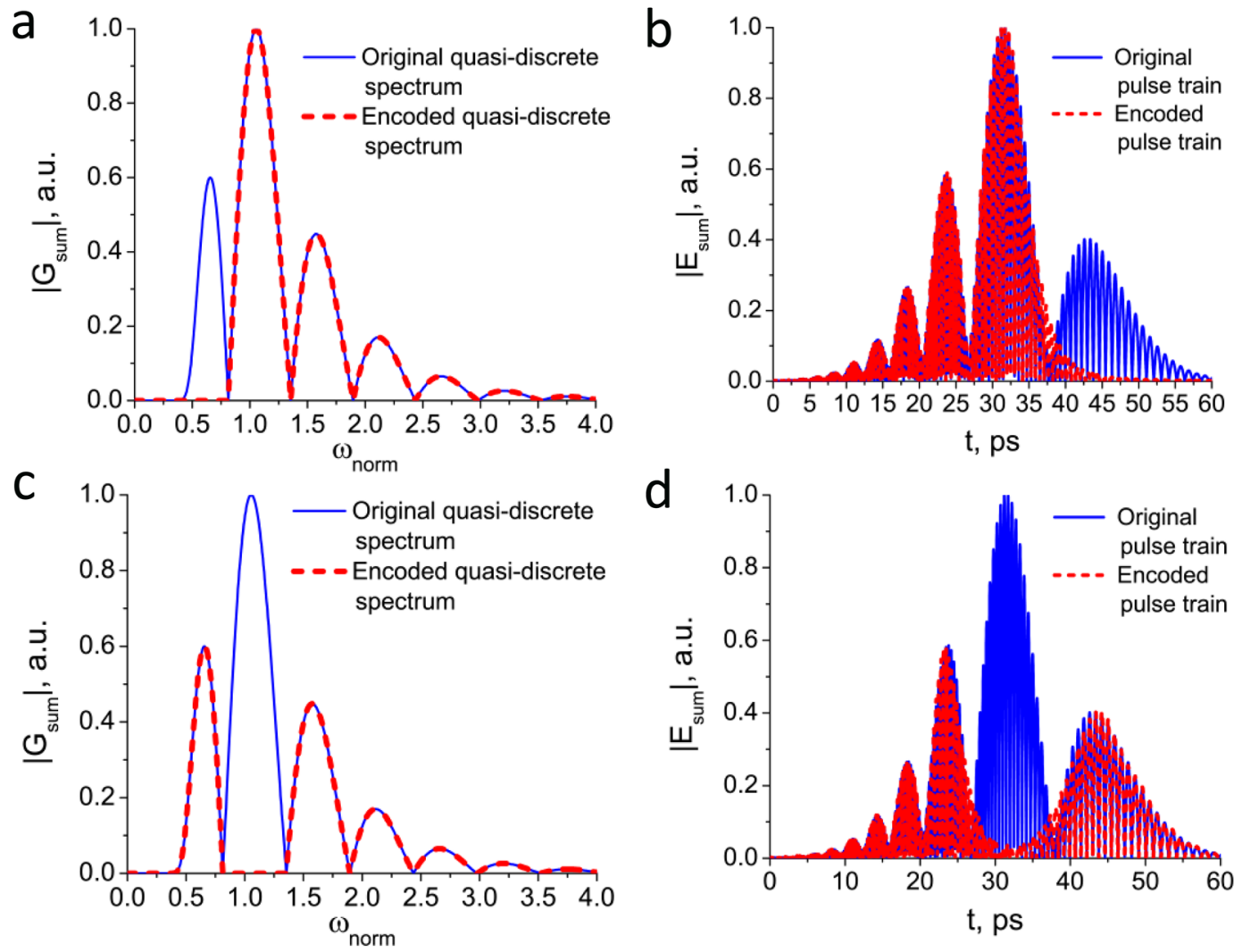

Figure 3. Comparison of original pulse (blue solid line) and encoded pulse (red dashed line) with cutting out one of the lines. (a), (c) Quasi-discrete spectrum and (b), (d) corresponding pulse train.

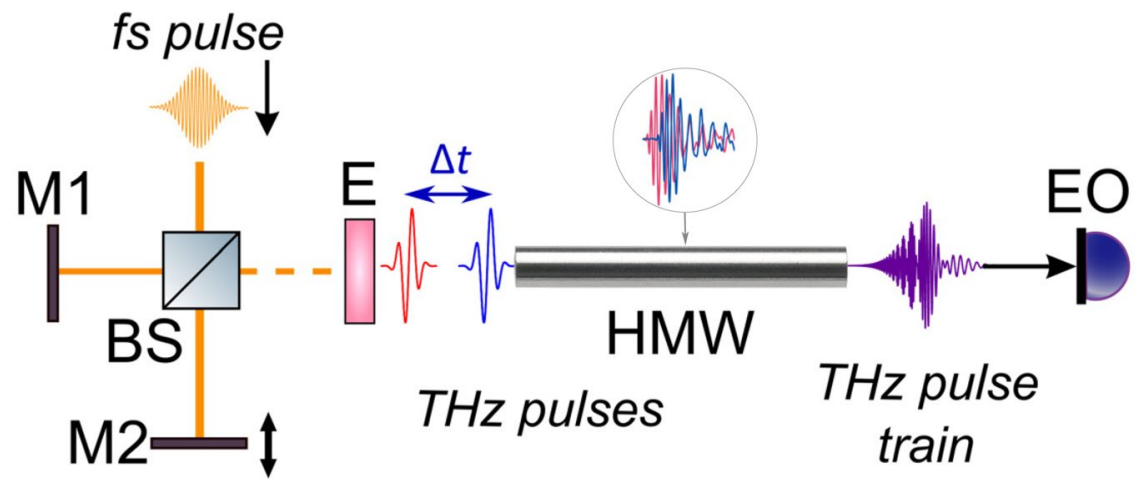

Figure 4. Experimental setup for $\mathrm{THz}$ pulse train generation from interference of two chirped $\mathrm{THz}$ pulses based on the conventional THz time-domain spectrometer. BS - beam splitter, M1, M2 - Michelson interferometer mirrors, E - THz emitter - InAs crystal for optical-to-THz conversion, HMW - hollow metal waveguide, $\mathrm{EO}$ - electro-optic detection system.

In order to further clarify the application prospects of this method, we provide the channel parameters (by channel we mean one of the peaks in the Fig. 2(c), e.g. the central one) of the original unoptimized communication system for the future establishment of the communication system. The ratio of the signal at the carrier frequency to noise $(\mathrm{C} / \mathrm{N})$ is of the order of $10^{3}$ or $30 \mathrm{~dB}$. BER in the case of binary message sources, bit rate of 75 $\mathrm{Mbit} / \mathrm{s}$ (which corresponds to the laser repetition rate of $75 \mathrm{MHz}$ for binary message sources) and spectral channel width of $250 \mathrm{GHz}$ is equal to $1.5 \cdot 10^{-7}$. For $50 \mathrm{~cm}$ transmission, the link budget is calculated to be: Received Power $(-94.7 \mathrm{dBm})=$ Transmitted Power $(-15.2 \mathrm{dBm})+$ Gains $(0 \mathrm{~dB})$ - Losses $(79.5 \mathrm{~dB})$. Competitive with existing short-range $\mathrm{THz} \mathrm{CW}$ channels $\left(10^{-3}-10^{-7} \mathrm{BER}\right)^{30-33}$, the method is promising in further establishment of the communication system.

In conclusion, this paper has shown experimentally the feasibility of forming a variable sequence of $\mathrm{THz}$ subpulses with $25-100 \mathrm{GHz}$ repetition rate in temporal domain. This is achieved by interference of two chirped $\mathrm{THz}$ 

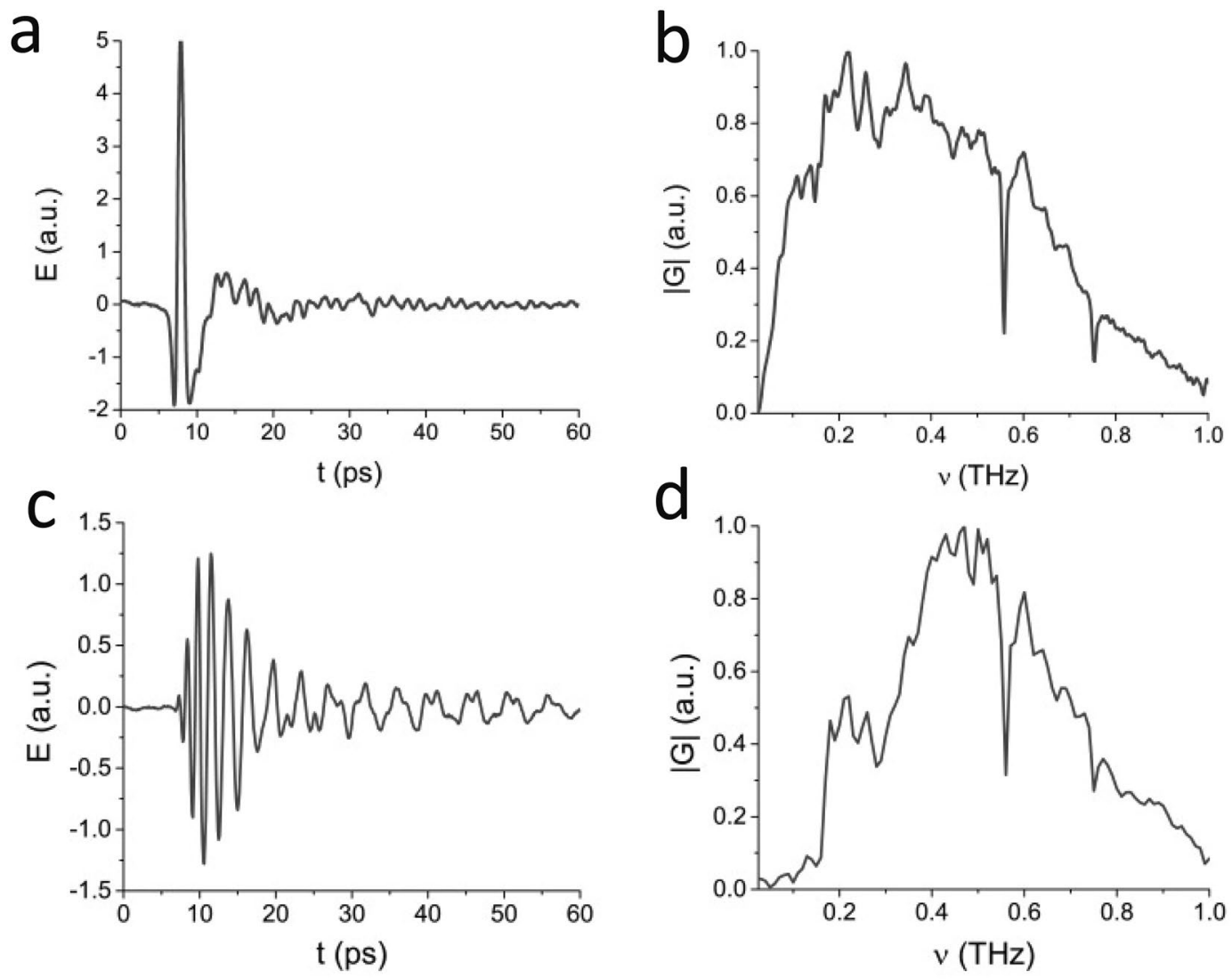

Figure 5. (a) Single THz pulse generated in InAs and (b) its spectrum. (c) The chirped THz pulse from hollow metal waveguide and (d) its spectrum.

pulses with a time delay less than their duration. As a result, the stable regular structure is formed in the spectral domain. The modulation frequency in the spectral and temporal structures is controlled by the time delay between the interfering pulses. Simulation-based results show that with the exponential chirped THz pulses, there is a "linkage relation" between the temporal and spectral structures. The data rate of such system is equal to the laser pulse repetition rate multiplied by the number of spectral peaks, in our case it is $75 \cdot 3=225 \mathrm{Mbit} / \mathrm{s}$. Estimated metrics of the communication channel based on the proposed method show that it is competitive with existing short-range $\mathrm{THz} \mathrm{CW}$ channels. Due to active research both in the field of integrated chip lasers ${ }^{35}$, as well as in the development of miniature $\mathrm{THz}$ antennas ${ }^{36,37}$, this method of modulation, control and coding of broadband $\mathrm{THz}$ radiation in the temporal and spectral domain can be further improved and applied for short-range $\mathrm{THz}$ communication.

\section{Methods}

Figure 4 illustrates the experimental setup for $\mathrm{THz}$ pulse train generation from interference of two chirped $\mathrm{THz}$ pulses based on the conventional THz time-domain spectrometer ${ }^{38}$. In this system, the $\mathrm{THz}$ radiation is generated due to the photo-Dember effect in an InAs crystal located in $2.4 \mathrm{~T}$ magnetic field ${ }^{39}$. The Yb-doped solid-state fs oscillator (central wavelength $1050 \mathrm{~nm}$, duration $100 \mathrm{fs}$, pulse energy $70 \mathrm{~nJ}$, repetition rate $75 \mathrm{MHz}$ ) is used as a pump. The THz radiation has central frequency $0.3 \mathrm{THz}$, estimated average power $30 \mu \mathrm{W}$, and FWHM $\sim 2$ ps (see Fig. 5(a,b)). [100]-oriented CdTe crystal is used for electro-optical detection.

It is known that two-beam interference leads to quasi-discrete spectrum ${ }^{11}$. In our work Michelson interferometer in front of a THz generator is used to create two consecutive fs pulses. One of the mirror is fixed while the other is located on the linear stage which allow to adjust the time delay $\Delta t$ between fs pulses. The time delay is chosen so that the $\mathrm{THz}$ pulses generated in the InAs crystal do not interfere with each other. Then these pulses pass through the hollow metal waveguide ${ }^{40}$, where they overlap in time and interfere (see inset of Fig. 4). As the result of the interference $\mathrm{THz}$ pulsed train and corresponding quasi-discrete spectrum are generated (see Fig. 1(b,c)). The time delay $\Delta t$ with the fixed waveguide parameters allows to control the modulation in the temporal and spectral structures ${ }^{25}$.

Figure 5(a,b) show example of generated single THz pulse and its spectrum and Fig. 5(c,d) illustrate the chirped $\mathrm{THz}$ pulse obtained from hollow stainless steel metal waveguide with $23 \mathrm{~mm}$ length, $0.89 \mathrm{~mm}$ tip inner diameter, and $1.43 \mathrm{~mm}$ outer diameter.

As can be seen from the Fig. 5, chirping in a metal waveguide leads to an increase in the $\mathrm{THz}$ pulse duration from 2 ps to $7 \mathrm{ps}$ (FWHM), while the corresponding spectrum shifts its central frequency to $0.45 \mathrm{THz}$ and 
undergoes some minor changes. For example, at low frequencies there is a drop due to the fact that at these frequencies the signal does not propagate in the waveguide with such parameters. However, the water absorption line at a frequency of $0.55 \mathrm{THz}$ remains unchanged. The $0.3 \mathrm{THz}$ dip is also caused by the propagation features of $\mathrm{THz}$ radiation in the waveguide ${ }^{29,40,41}$.

Received: 14 February 2020; Accepted: 21 May 2020;

Published online: 11 June 2020

\section{References}

1. Andrews, J. G. et al. What will 5g be? IEEE J. on selected areas communications 32, 1065-1082 (2014).

2. Zhang, Z. et al. $6 \mathrm{~g}$ wireless networks: Vision, requirements, architecture, and key technologies. IEEE Veh. Technol. Mag. 14, 28-41 (2019).

3. Calvanese Strinati, E. et al. $6 \mathrm{~g}$ : The next frontier: From holographic messaging to artificial intelligence using subterahertz and visible light communication. IEEE Veh. Technol. Mag. 14, 42-50 (2019).

4. Busari, S. A., Huq, K. M. S., Mumtaz, S. \& Rodriguez, J. Terahertz massive mimo for beyond-5g wireless communication. In ICC 2019 - 2019 IEEE International Conference on Communications (ICC), 1-6 (2019).

5. Ummethala, S. et al. Thz-to-optical conversion in wireless communications using an ultra-broadband plasmonic modulator. Nat. Photonics 13, 519-524 (2019).

6. Huq, K. M. S. et al. Terahertz-enabled wireless system for beyond-5g ultra-fast networks: A brief survey. IEEE Netw. 33, 89-95 (2019).

7. Rappaport, T. S. et al. Wireless communications and applications above $100 \mathrm{ghz}$ : Opportunities and challenges for $6 \mathrm{~g}$ and beyond. IEEE Access 7, 78729-78757 (2019).

8. Cacciapuoti, A. S., Sankhe, K., Caleffi, M. \& Chowdhury, K. R. Beyond 5g: Thz-based medium access protocol for mobile heterogeneous networks. IEEE Commun. Mag. 56, 110-115 (2018).

9. Boulogeorgos, A.-A. A. et al. Terahertz technologies to deliver optical network quality of experience in wireless systems beyond $5 \mathrm{~g}$. IEEE Commun. Mag. 56, 144-151 (2018).

10. Delfyett, P. J. et al. Advanced ultrafast technologies based on optical frequency combs. IEEE J. Sel. Top. Quantum Electron. 18, 258-274 (2012)

11. Alfano, R. R. \& Zeylikovich, I. Method and apparatus for producing a multiple optical channel source from a supercontinuum generator for wdm communication US Patent 7,245,805 (2007).

12. Lu, X. \& Zhang, X.-C. Investigation of ultra-broadband terahertz time-domain spectroscopy with terahertz wave gas photonics. Front. Optoelectronics 7, 121-155 (2014)

13. Li, H. et al. Dynamics of ultra-broadband terahertz quantum cascade lasers for comb operation. Opt. Express 23, 33270-33294 (ts).

14. Liu, P., Xu, D. \& Yao, J. Analysis on characteristic and application of thz frequency comb and thz sub-comb. In Journal of Physics: Conference Series, vol. 276, 012218 (IOP Publishing, 2011).

15. Udem, T., Holzwarth, R. \& Hänsch, T. W. Optical frequency metrology. Nature 416, 233-237 (2002).

16. Delfyett, P. J. et al. Optical frequency combs from semiconductor lasers and applications in ultrawideband signal processing and communications. J. Light. Technol. 24, 2701 (2006).

17. Kleine-Ostmann, T. \& Nagatsuma, T. A review on terahertz communications research. J. Infrared, Millimeter, Terahertz Waves 32, 143-171 (2011).

18. Schneider, T., Wiatrek, A., Preußler, S., Grigat, M. \& Braun, R.-P. Link budget analysis for terahertz fixed wireless links. IEEE Transactions on Terahertz Sci. Technol. 2, 250-256 (2012).

19. Piesiewicz, R. et al. Short-range ultra-broadband terahertz communications: Concepts and perspectives. IEEE Antennas Propag. Mag. 49, 24-39 (2007).

20. Han, C., Bicen, A. O. \& Akyildiz, I. F. Multi-wideband waveform design for distance-adaptive wireless communications in the terahertz band. IEEE Transactions on. Signal Process. 64, 910-922 (2015).

21. Lin, C. \& Li, G. Y. Adaptive beamforming with resource allocation for distance-aware multi-user indoor terahertz communications. IEEE Transactions on Commun. 63, 2985-2995 (2015).

22. Han, C. \& Akyildiz, I. F. Distance-aware bandwidth-adaptive resource allocation for wireless systems in the terahertz band. IEEE Transactions on Terahertz Sci. Technol. 6, 541-553 (2016).

23. Dunn, A. et al. High-speed modulation of a terahertz quantum cascade laser by coherent acoustic phonon pulses. Nat. Commun. 11, $1-8(2020)$.

24. Grachev, Y. V. et al. Wireless data transmission method using pulsed thz sliced spectral supercontinuum. IEEE Photonics Technol. Lett. 30, 103-106 (2017).

25. Tsypkin, A. N., Putilin, S. E., Okishev, A. V. \& Kozlov, S. A. Ultrafast information transfer through optical fiber by means of quasidiscrete spectral supercontinuums. Opt. Eng. 54, 1-3 (2015).

26. Tcypkin, A. \& Putilin, S. Spectral-temporal encoding and decoding of the femtosecond pulses sequences with a thz repetition rate. Appl. Phys. B 123, 44 (2017).

27. Melnik, M., Tcypkin, A., Putilin, S., Kozlov, S. \& Rodrigues, J. J. Analysis of controlling methods for femtosecond pulse sequence with terahertz repetition rate. Appl. Phys. B 125, 98 (2019).

28. Kozlov, S. A., Drozdov, A. A., Choudhary, S., Kniazev, M. A. \& Boyd, R. W. Suppression of self-focusing for few-cycle pulses. J. Opt. Soc. Am. B 36, G68-G77 (2019).

29. Gallot, G., Jamison, S. P., McGowan, R. W. \& Grischkowsky, D. Terahertz waveguides. J. Opt. Soc. Am. B 17, 851-863 (2000).

30. Moeller, L., Federici, J. \& Su, K. Thz wireless communications: $2.5 \mathrm{gb} / \mathrm{s}$ error-free transmission at 625 ghz using a narrow-bandwidth $1 \mathrm{mw}$ thz source. In 2011 XXXth URSI General Assembly and Scientific Symposium, 1-4 (2011).

31. Ducournau, G. et al. $32 \mathrm{gbit} / \mathrm{s}$ qpsk transmission at $385 \mathrm{ghz}$ using coherent fibre-optic technologies and thz double heterodyne detection. Electron. Lett. 51, 915-917 (2015).

32. Jia, S. et al. Thz wireless transmission systems based on photonic generation of highly pure beat-notes. IEEE Photonics J. 8, 1-8 (2016).

33. Zhao, M., Zhou, W. \& Yu, J. 3.5 gbit/s ook thz signal delivery over $88 \mathrm{~cm}$ free-space at 441.504 ghz. Microw. Opt. Technol. Lett. 60, 1435-1439 (2018).

34. Mendis, R. Nature of subpicosecond terahertz pulse propagation in practical dielectric-filled parallel-plate waveguides. Opt. Lett. 31, 2643-2645 (2006).

35. Rafailov, E. U., Cataluna, M. A. \& Sibbett, W. Mode-locked quantum-dot lasers. Nat. photonics 1, 395-401 (2007).

36. Barr, P., Zaman, A. \& Miranda, F. A compact, broadband antenna for planetary surface-to-surface wireless communications. Microw. Opt. Technol. Lett. 48, 521-524 (2006).

37. Han, S.-P. et al. Compact fiber-pigtailed ingaas photoconductive antenna module for terahertz-wave generation and detection. Opt. express 20, 18432-18439 (2012). 
38. Grachev, Y. V., Osipova, M. O. \& Bespalov, V. G. Comparison of an electro-optical system and photo-conducting antenna employed as detectors of pulsed terahertz radiation by means of a new method for measuring spectral width. Quantum Electron. 44, 1170-1172 (2014).

39. Bespalov, V. et al. Methods of generating superbroadband terahertz pulses with femtosecond lasers. J. Opt. Technol. 75, 636-642 (2008).

40. McGowan, R. W., Gallot, G. \& Grischkowsky, D. Propagation of ultrawideband short pulses of terahertz radiation through submillimeter-diameter circular waveguides. Opt. Lett. 24, 1431-1433 (1999).

41. Nazarov, M. M. et al. Eight-capillary cladding thz waveguide with low propagation losses and dispersion. IEEE Transactions on Terahertz Sci. Technol. 8, 183-191 (2018).

\section{Acknowledgements}

This work was partially supported by RFBR according to the research project No. 17-00-00275 (17-00-00272), by Ministry of Science and Higher Education of the Russian Federation project "Extreme phenomena and coherent structures in nonlinear physics", by FCT/MCTES through national funds and when applicable cofunded EU funds under the Project UIDB/EEA/50008/2020, and by Brazilian National Council for Research and Development (CNPq) via Grants No. 431726/2018-3 and 309335/2017-5. The authors acknowledge Dr. M. Nazarov from NRC «Kurchatov Institute» and Dr. M. Song from ITMO University for fruitful discussions.

\section{Author contributions}

X.L. and M.Z. analysed the results, M.M. performed numerical simulations, E.O. and A.T. conducted the experiment, J.R. prepared review of the existing works in the field of study, S.K. conceived the experiment. All authors reviewed the manuscript.

\section{Competing interests}

The authors declare no competing interests.

\section{Additional information}

Correspondence and requests for materials should be addressed to M.Z.

Reprints and permissions information is available at www.nature.com/reprints.

Publisher's note Springer Nature remains neutral with regard to jurisdictional claims in published maps and institutional affiliations.

(c) (i) Open Access This article is licensed under a Creative Commons Attribution 4.0 International License, which permits use, sharing, adaptation, distribution and reproduction in any medium or format, as long as you give appropriate credit to the original author(s) and the source, provide a link to the Creative Commons license, and indicate if changes were made. The images or other third party material in this article are included in the article's Creative Commons license, unless indicated otherwise in a credit line to the material. If material is not included in the article's Creative Commons license and your intended use is not permitted by statutory regulation or exceeds the permitted use, you will need to obtain permission directly from the copyright holder. To view a copy of this license, visit http://creativecommons.org/licenses/by/4.0/.

(c) The Author(s) 2020 